\title{
Electronic Spectroscopy and Photoionization of LiBe
}

Thomas D. Persinger, Jiande Han, and Michael C. Heaven*

Department of Chemistry, Emory University, Atlanta, GA 30322

* Corresponding author.

Email: mheaven@emory.edu

Phone: 4047276617

\section{Supplementary Information}




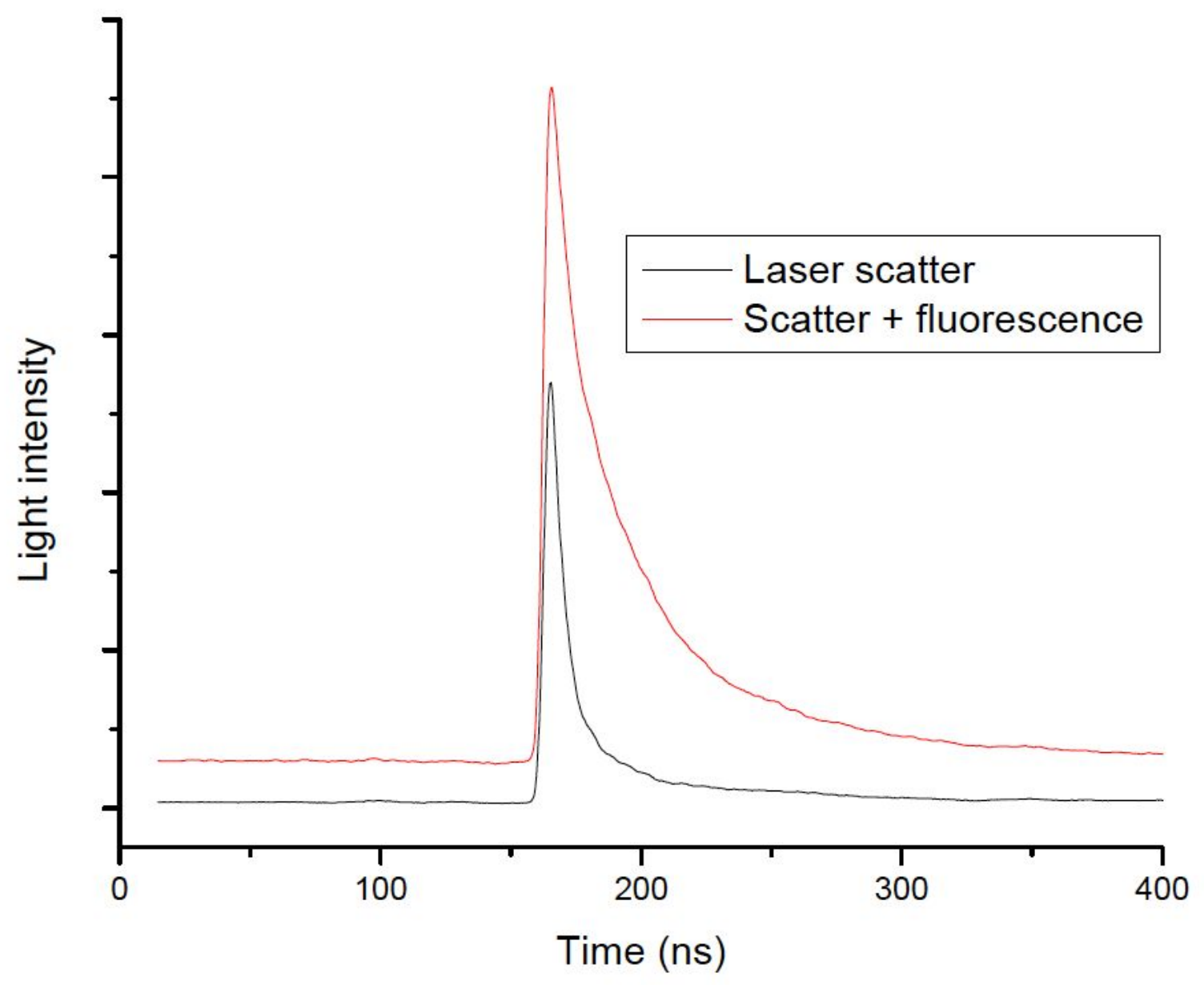

Fig. S1a. Fluorescence and scattered laser light signals associated with excitation of the LiBe $2^{2} \Pi-X^{2} \Sigma^{+} 2-0$ band. The red trace was recorded with production of LiBe by laser ablation. The black trace, which shows only scattered laser light, was recorded with the ablation laser blocked to prevent the formation of LiBe. 


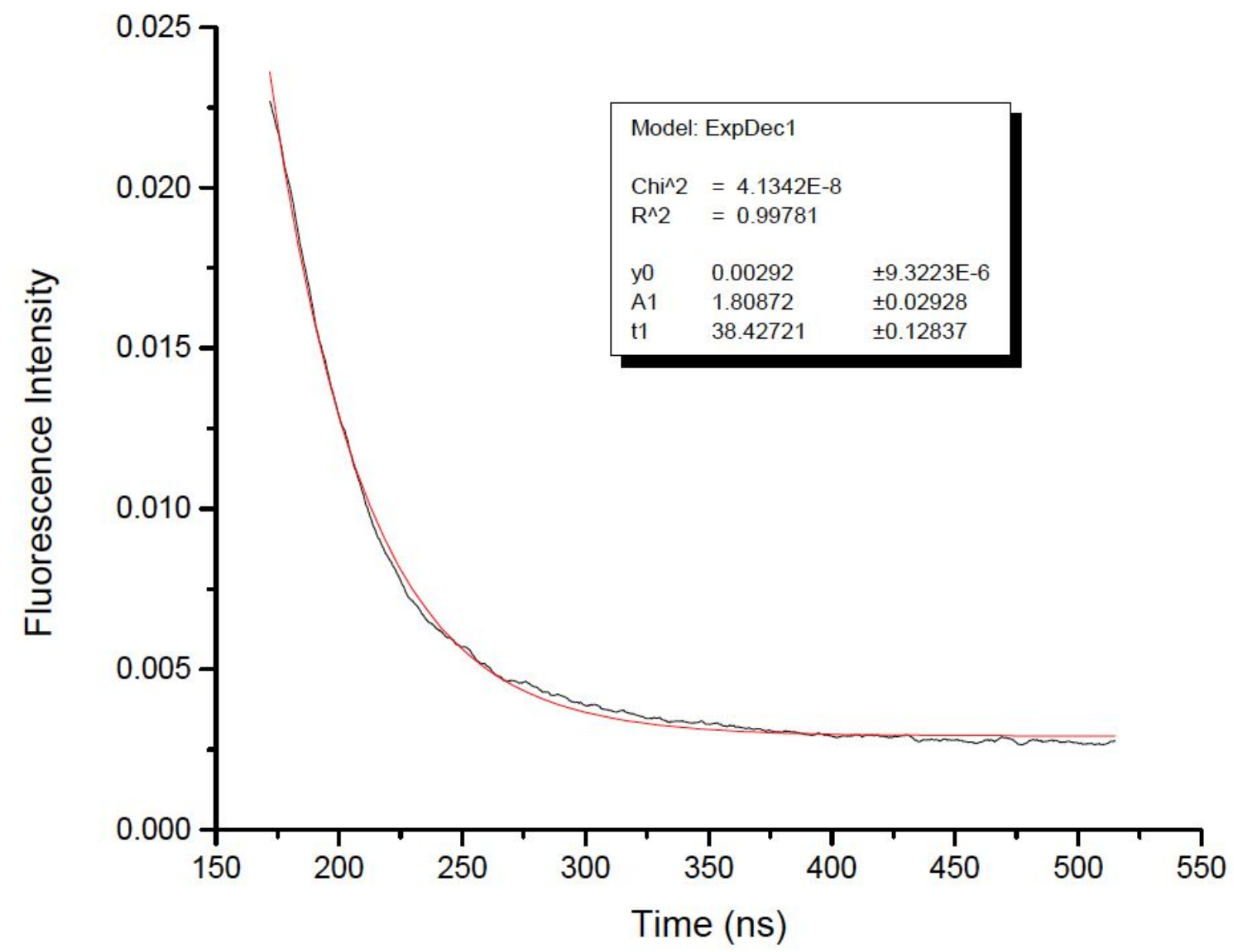

Fig. S1b. Time resolved fluorescence from $\mathrm{LiBe} 2^{2} \Pi, v^{\prime}=2$. The experimental data (black trace) were obtained by the subtraction of the curves shown in Fig S1a. The red trace is a non-linear least-squares fit to the decay curve. This fit yields a spontaneous decay lifetime of $38.4 \mathrm{~ns}$. 


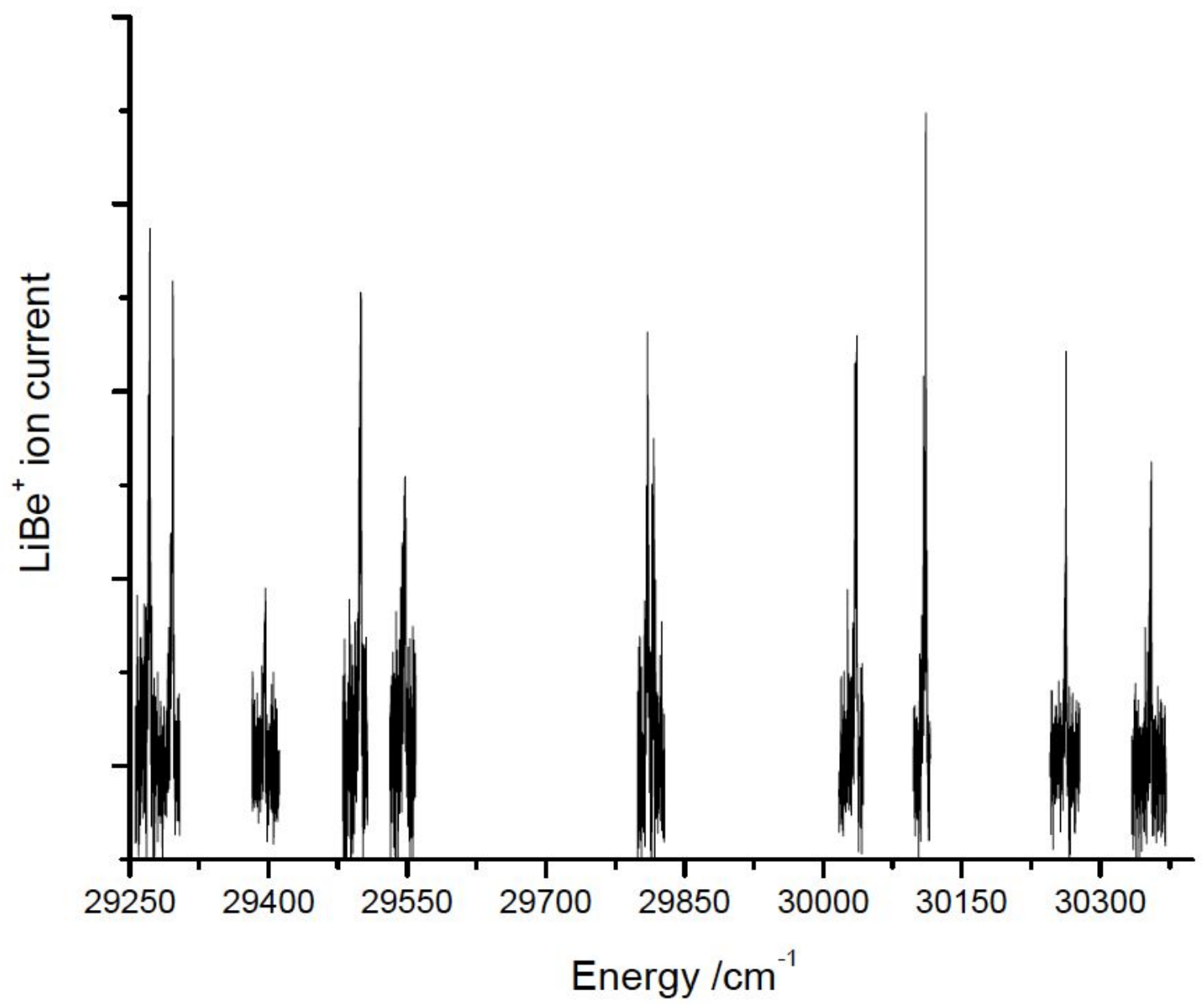

Fig. S2. Low-resolution RE2PI spectrum of LiBe in the near UV spectral range. This trace was recorded using one-color, two-photon ionization. 


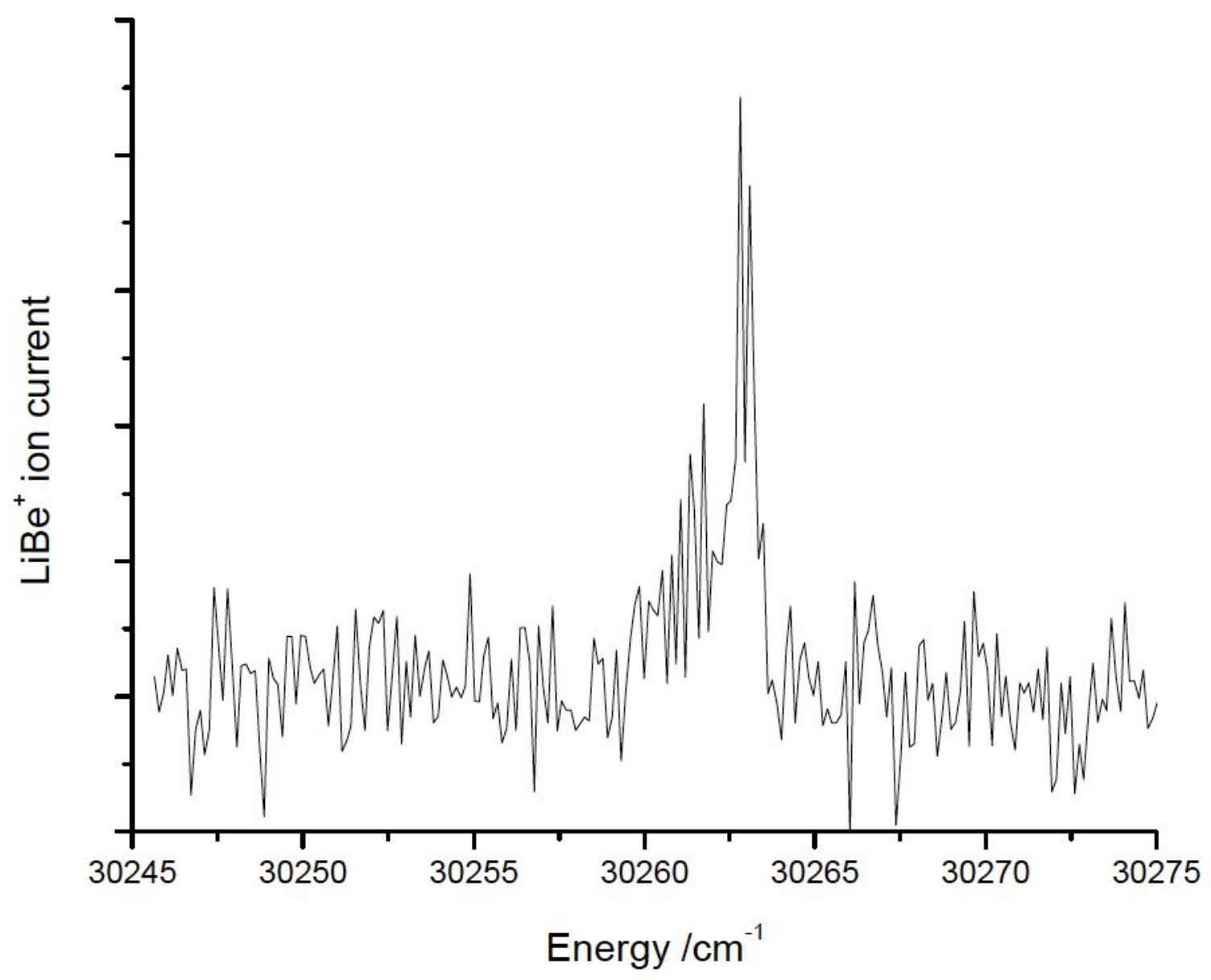

Fig. S3. Expanded view of the LiBe band at $30262 \mathrm{~cm}^{-1}$. It can be seen that the rotational band contour is red shaded, but the detailed rotational structure is obscured by the noise. Similar contours were observed for the bands collected in Fig. S1. 